\section{Troubleshooting Forum}

\section{Molecular Biology Techniques Q\&A}

\author{
qPCR: avoiding signals in the no-template control
}

This month's question from the Molecular Biology Forums (online at molecularbiology. forums.biotechniques.com) comes from the "Real-time qPCR/qRT-PCR Methods" section. Entries have been edited for concision and clarity. Mentions of specific products and manufacturers have been retained from the original posts, but do not represent endorsements by, or the opinions of, BioTechniques.

\section{How can I avoid technical replicate variation and signals in my no-template controls? (Thread 21619)}

I am having some problems with qPCR using plant RNA. I see amplification in the no-template control (NTC) reactions and unacceptable technical replicate differences in the $\mathrm{Ct}$ values.

I tried total RNA extraction and using Dynabeads from Invitrogen and prefer the latter because I get better-quality RNA as determined by Nanodrop and Bioanalyzer. I reversetranscribe using Superscript II and oligo(dT)20. I am using the MX3000P system from Stratagene and SYBR Green II. The genes form secondary structures and the genome is duplicated. Because of this, I was very careful with the primer design and tried several primer dilutions. Adding 2.5\% DMSO helped very little and using more DMSO inhibited the reaction. I checked every step on a gel and sequenced the PCR products. I verified that there isn't genomic DNA contamination by PCR with suitable primers. I guess the variability is coming from poor-quality cDNA. How can I solve this problem?

A The amplification you are seeing may actually be primer dimer and not real amplification. Primer dimers still have melting peaks. If this is your problem, you might try reducing the amount of primer you use.

If the problem is with the cDNA as you suspect, you could try using a mix of random primers and oligo(dT). Qiagen supplies this option with the QuantiTect reverse transcription enzyme and Bio-Rad offers it with their iScript enzyme.

A Unacceptable replicates are usually caused by pipetting errors. ROX dye should normalize for differences in the mastermix. I get very tight replicates using the Qiagen SYBR green mastermix.

Is your instrument calibrated and running properly?

Q I'm using a ROX dye. It helps a little in correcting the signal, but the results aren't affected much by its presence. I see melting curves for the ROX channel, the NTC, and the NTC SYBR Green signal.

I tried running a few plates on an Opticon machine but got similar results. The instruments are monitored by the technician at the core facility and other users haven't reported problems. I tried running the genes in different positions on the plate and still got variable results, so it doesn't seem to be a problem with the machine.

A The ROX channel cannot have a melting peak. The melting peak is caused by the change in fluorescence as SYBR green binds dsDNA. As the temperature goes up and melts the strands, the SYBR is released and stops fluorescing. ROX is not going to do that since it doesn't bind DNA. If you see a peak in your water control, then you have contamination. That could cause variability if different samples have varying levels of contamination.

After you make a batch of cDNA, do you dilute it for qPCR or do you dilute the RNA and then perform the cDNA reactions? I recommend the first way.

Vol. $50 \mid$ No. $4 \mid 2011$

\section{Do you do cell research?}

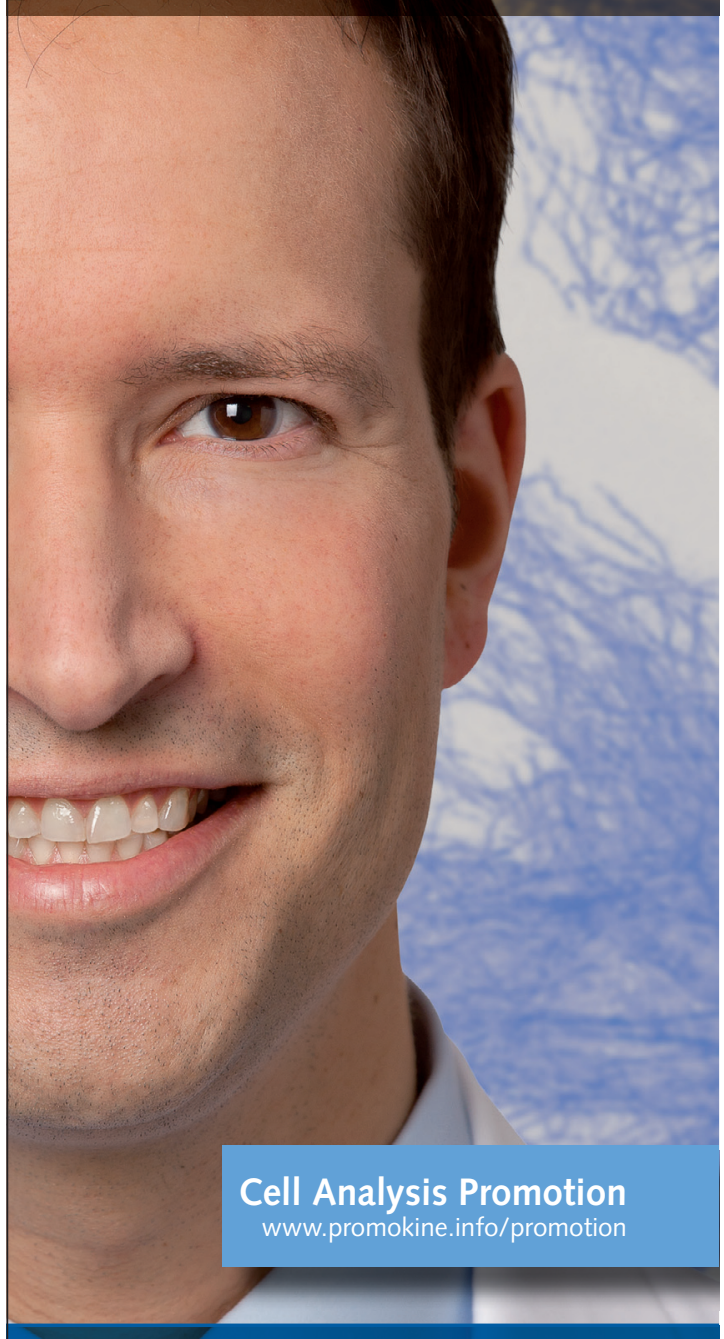

\section{Go for PromoKine !} Your cell research partner

- Cell Analysis Products

e.g. Cell Viability \& Cytotoxicity Kits

- Apoptosis Detection Kits

e.g. Caspase \& Annexin V Kits

Also Transfection Reagents, Cytokines, Mycoplasma Elimination, and much more!

\section{www.promokine.info}

\section{PromoKine}

PromoCell GmbH North America: 1 - 866 - 251 - 2860 (toll free) Deutschland: 0800 - 7766623 (gebührenfrei) United Kingdom: 0800 - 960333 (toll free) France: 0800909332 (ligne verte) Other countries: +496221 - 649340 
Q I don't dilute the RNA, but instead reverse-transcribe and then dilute the cDNA.

A Your NTC signal might only be from the primer-dimer product, so it may actually be negative. It is common to see dimers form when there is no actual template for the primers to work on. You may also see this in the high-dilution wells since there is little template. Right after annealing, you can add another data acquisition step at a higher temperature than the extension step. That should melt the dimers so that you only read specific signals.

If your contamination is a PCR product, you can try using dUTP in the $\mathrm{qPCR}$ mix. With dUTP, the PCR product will have U incorporated into the strand. That allows you to perform a 2-min digest with an uracil-N-glycosylase before activating the Taq DNA polymerase. Any contaminating PCR products will be digested.

A Phenolics and possibly polysaccharides in your original plant extracts can carry over into your RNA samples and hurt the efficacy of your RT reactions.

Q I added an extra fluorescence reading step at $81^{\circ} \mathrm{C}$ after elongation that eliminated most of the signal in the NTC amplifications.

I wonder if the slightly different melting temperatures are caused by secondary structures or folding in the cDNA that prevents consistent amplification of my products.

A You might want to explore the possibility of splice variants of your targets of interest. Are you sure of your target sequences?

A Invitrogen has a reverse transcriptase called Thermoscript that can be used at higher temperatures to help remove secondary structure. Qiagen reverse transcriptase reads through secondary structures.

A It is documented in papers by S.A. Bustin (2002, J. Mol. Endocrinol. 29:23-39) and Wong and Medrano (2005, BioTechniques 39:75-85) that the biggest source of variation in qPCR is the person doing the experiment. You may be missing something simple like proper mixing before dispensing into the reaction tubes. Plates and tubes are very prone to cross-contaminations. I would suggest leaving empty wells around your NTCs and vortexing everything.

A I agree with the previous comment: vortexing reagents before use is key. White-welled plates from Eppendorf have higher reflectivity, so they give higher signal, tighter replicate Cts, and are cheaper than other plates. The only drawback is that it is harder to see the wells where you have already dispensed your reagents.

A You might also try autoclaving your diluent solution again. Reagent contamination is a chief cause of NTC signal and usually the suspect reagent is the diluent water or TE.

A You might want to put your samples into maximum recovery tubes or use a carrier nucleic acid. Or you could try another type of detection like TaqMan.

A You might avoid primer dimer signals by setting the detection temperature between the melting temperature of the dimer and the melting temperature of the target.

The most likely causes of your variable results in the replicates are that the plate is not properly sealed, your target gene expression is low (Ct 35-40, dynamic range with Mx3000P or similar instruments is poorer than with ABI7900), or the quality of your RNA is not good.

Selected and edited by Kristie Nybo, Ph.D.

BioTechniques 50:213-215 (April 2011)

doi 10.2144/000113648

To purchase reprints of this article, contact: biotechniques@fosterprinting.com

\section{The days of comparing apples to apples are over!
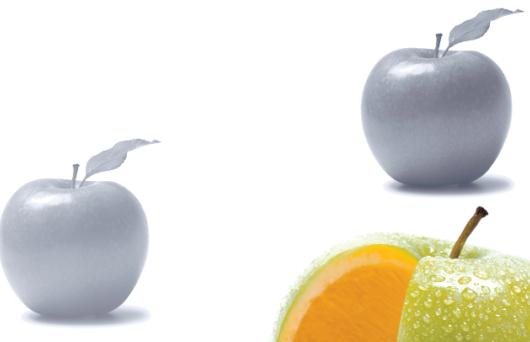 \\ Introducing Universal Mycoplasma Detection.}

\section{Not all PCR-based kits are the same.}

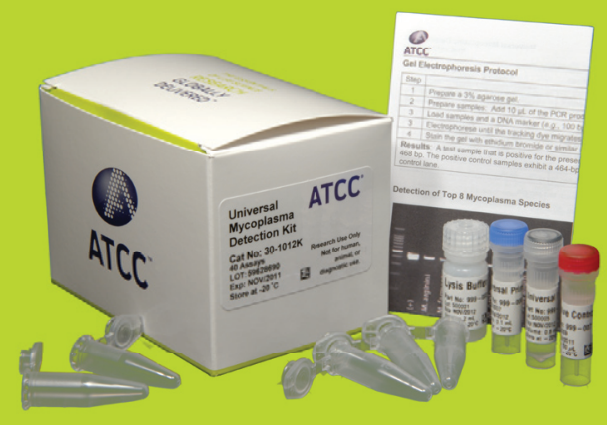

- Superior sensitivity

- Confirmed specificity

- Broad detection range

- Results in less than 3 hours

See for yourself:

www.atcc.org/MDK1 